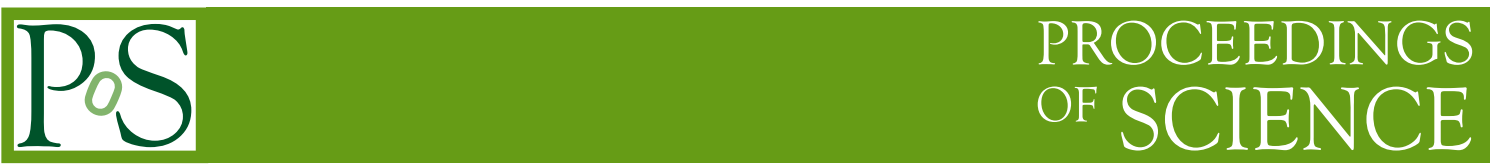

\title{
The ridges and the cone as "QGP corona" effects
}

\author{
Edward Shuryak \\ Department of Physics and Astronomy \\ University at Stony Brook 11794 NY, USA \\ E-mail: shuryaketonic.physics.sunysb.edu
}

\begin{abstract}
Two experimentally observed structures, called a "cone" and a "hard ridge", have been discovered in dihadron correlation function with large- $p_{t}$ trigger, while "soft ridge" is a similar structure seen without hard trigger. All three can be viewed as traces left by a moving charge in matter, on top of overall expansion. A puzzle is why those perturbations are apparently rather well preserved till the time of the fireball freezeout, instead of spreading out with the speed of sound. The proposed answer is related to new "magnetic" picture of the near- $T_{c}$ matter, also known as M-phase. Because of small density of free quarks the electric screening mass is also small, thus we argue the (gluo)electric field remains in it for the duration of the process. The consequence is existence of a "QGP corona", not dissimilar to that of the Sun, in which electric fields influence propagation of perturbations and even form metastable flux tubes. The natural tool for its description is (dual) magnetohydrodynamics, which predicts that instead of sound there are two modes, one with larger and one with smaller velocity. The latter can be zero, hinting for formation of pressure-stabilized flux tubes. We argue that those flux tubes are related with cone and ridges. We also find that, contrary to naive expectations, near $T_{c}$ these tubes (or color strings) should have tighter tension and longer life time than in vacuum: this agrees with PHOBOS clustering data. At the end we discussed expected dependence of ridges and cone on the collision energy, in connection to RHIC energy scan run.
\end{abstract}

5th International Workshop on Critical Point and Onset of Deconfinement - CPOD 2009,

June 08 - 122009

Brookhaven National Laboratory, Long Island, New York, USA 


\section{Introduction}

As one of the originators of this series of conferences, I spoke at all of them. In view of coming low energy run of RHIC it was tempting to discuss again the hydrodynamical signals of the onset of deconfinement. Indeed, as the line of the initial excitation parameterized by collision energy $\left(T_{i n}(s), \mu_{i n}(s)\right)$ reaches the phase transition line, and then at some other energy goes out of it into the QGP, those should give produce observable changes in radial, dipole and elliptic flows. Unfortunately, these effects, known collectively as signals of the "softest point", have not received enough attention as compared to the signals of the critical point. And yet I hope those are known well enough and will get attention in the analysis of flows in that run.

This talk is about entirely different aspect of "onset of deconfinement", based on quite recent development in understand of the "magnetic side of QGP". While confinement itself is long believed to be due to "dual superconductor" [1] made of Bose-condensed magnetically charged objects (color monopoles), recent activity $[2,3,4,5]$ concentrated on the temperature interval at and right above $T_{c}$ where these monopoles are "normal" (not Bose-condensed) component of the plasma. Furthermore, at $T=(0.9 . .1 .4) T_{c}$ those monopoles even are dominant, while electric components - quarks and gluons - are suppressed. The idea that one kind of charges is dominant is the central point of this development: it leads to the conclusion that electric fields should remain unscreened. If so, there should be a "QGP corona" with many spectacular phenomena, which can be described by the dual magnetohydrodynamics (DMHD) instead of the usual hydro.

It is based on my recent paper [6] devoted to propagation of perturbation on top of hydrodynamically expanding fireball. It discusses two scenarios, both leading to conclusion that they may get split into two modes, propagating with very different speed. For lack of space we will not discuss here the "acoustic scenario" in which the secondary waves are induced by the sharply dropping speed of sound in the M-phase. However, as it was found that it requires too sharp drop in a speed of sound, not supported by current lattice data.

We will instead focus on the second scenario on this paper which also leads to double cones, now as two components of Alfven waves in a (dually)magnetized medium. Furthermore, some of them have small or even zero expansion velocity, and indication to existence of stabilized electric flux tubes in near-Tc temperature interval. Metastable microscopic flux tubes in the near- $T_{c}$ region had also been considered in a different context before, by Liao and myself $[7,4]$ in connection with lattice data on lattice potentials and charmonium survival. Yet again, although such tubes have good reasons to exist, the final conclusion on whether they are robust enough to explain the observed "cone" and two "ridges" would require a lot of further experimental and theoretical work.

\section{The magnetic side of QGP}

In 2006 the so called "magnetic scenario" [2,3] has been proposed, which basically views the near- $T_{c}$ region, from about $0.8 T_{c}$ up to $1.4 T_{c}$ as magnetic plasma, dominated by (gluomagnetic) monopoles. Recent theory developments along this line lead to understanding of enhanced transport cross section $[2,8]$ (in classical and quantum settings respectively) explaining why matter at RHIC is such a good liquid and making predictions for the LHC. Its main reason is the so called "caging" phenomenon, induced by the Lorentz force. Magnetic sector is a liquid, as seen on the 

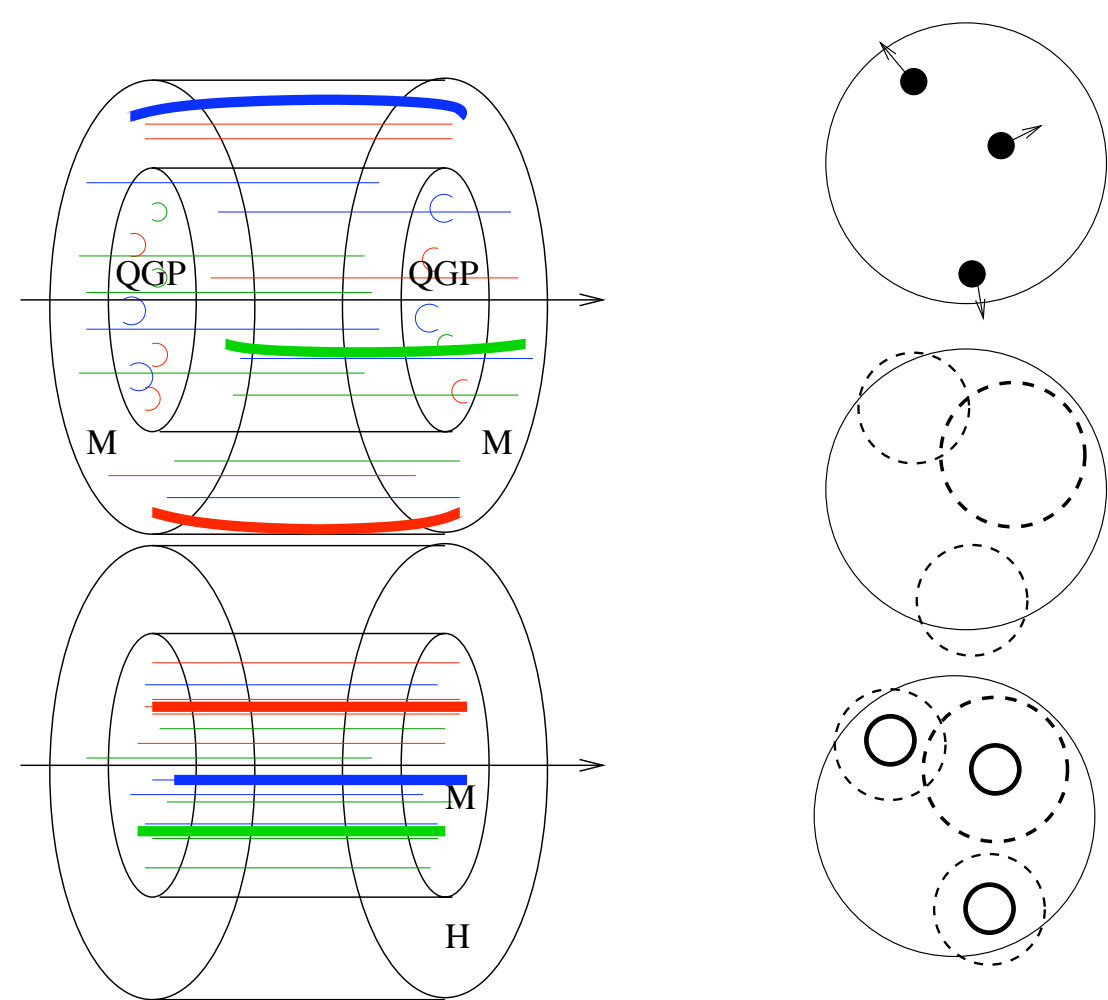

Figure 1: (left)A snapshot of unscreened electric (dual-magnetic) field in the $M$ (near- $T_{c}$ ) region of the fireball. Upper and lower ones correspond to full RHIC energy and the reduced energy (analogous to SPS). (right)A sketch of the transverse plane of the colliding system: the "spots" of extra density (a) are shown as black disks, to be moved by collective radial flow (arrows). Naive sound expansion (b) would produce large-size and small amplitude wave: yet the correct solution includes also brighter secondary wave (c) of smaller radius.

lattice in spatial correlators of monopoles [5], and the magnetic coupling does run in the direction opposite to electric one, as predicted in [2].

What is important for the present talk is the the behavior of the electric and magnetic screening masses. Multiple lattice studies, e.g. ref [9] have shown that at $T<1.4 T_{C}$ the relation between electric and magnetic screening masses get inverted, $M_{M}>M_{E}$. Furthermore, at $T \rightarrow T_{c}$ the density of electric objects (quarks and gluons) is very strongly suppressed, both by their heavy effective masses and small Polyakov loop expectation value $\langle L\rangle$, going to zero below $T_{c}$. Thus one has $M_{M}\left(T \rightarrow T_{c}\right) \approx 3 T_{c}, M_{E}\left(T \rightarrow T_{c}\right) \approx 0$. It means that in the M-phase of the collision magnetic field is well screened while electric one remains unscreened for a significant time.

This leads to the central idea: that QGP produced in central part of RHIC collisions should have a "dual corona" in which electric (rather than magnetic) fields coexist with the plasma, affecting both the overall expansion and the propagation of perturbations. We will suggest to use "dual magnetohydrodynamics" (DMHD) for the description of diffuse electric fields in the M-phase, in particular study their effect on the velocity of propagation of small perturbations. We will further argue below that like solar corona, that of QGP should have metastable flux tubes, although microscopically thin ones which cannot be directly described by DMHD approximation.

In a magnetic plasma, one expects existence of electric flux tubes. They are similar to those which confine electric charges in the vacuum $(\mathrm{T}=0)$, with a "coil" made of magnetic particles 
rotating around the electric flux. However, as proposed by Liao and myself [7, 4], those can also exist at $T>T_{c}$. As the current is not a supercurrent, they are not stable forever but are metastable. Perhaps surprising to many, those flux tubes are tighter (have larger tension) and seeming longer lived than the vacuum ones.

The original indications for their existence came from lattice data on finite-T potentials. In brief, the central observation is large difference between the free energy $F(T, r)$ and the potential energy

$$
V(T, r)=F(T, r)+T S(T, r)
$$

associated with quark pair at distance $r$. Unlike $F$, both $V$ and $S$ have linear (with $r$ ) which peaks at $T_{c}$ and disappear only at $T \sim 1.4 T_{c}$. The physical difference between the two, first discussed by Zahed and myself [10] in the context of hadronic spectroscopy at finite $T$, is that $F$ corresponds to adiabatically slow motion of the quarks, slow enough to produce maximal entropy possible and reach thermal equilibrium at any $r$. However when quarks are moving with certain velocity $v$ away from each other, only a fraction $x$ of the maximal entropy can be produced (because of LandauZener argument on level crossing): thus the effective potential would be

$$
V_{e f f}(T, r)=F(T, r)+(1-x) T S(T, r)
$$

For relatively rapid motion in which no entropy is produced, $x=0$, and one returns to $V(T, r)$. These arguments have been important to discussion of charmonium survival at RHIC.

The difference between the two potentials is discussed in detail in [4]: in short $F(T, r)$ has been related to "supercurrent coil" and $V(T, r)$ to "normal metastable coil". If so, large peak at $T_{c}$ of the tension of $V(T, r)$ is related [7, 4] to a peak in "normal" monopole density at $T_{c}$. The condensed and normal monopole density needed to accommodate flux tubes with such tensions have been derived in Refs[7, 4] and compared with direct lattice observations of monopoles. The summary of those studies is that metastable flux tubes seem to exist at $T<1.4 T_{c}$, changing from stable to metastable around $T_{c}$.

\section{QGP corona}

The word "corona" used here comes from the physics of the Sun. Let me briefly remind the reader that it was started by Galileo Galilei, who in 1612 spent some time observing the motion of the black spots on the Sun and correctly concluded from motion of the spots that they must resign on a surface of a rotating sphere: he thus argued the spots were not shadows of some planets passing in front, as it was thought of before. In due time relation between the spots and solar magnetism was understood: modern telescopes allows one to see the fine structure of solar spots, resolving individual magnetic flux tubes. Better understanding of solar magnetism came with the advance of plasma physics in 1940's and development of MHD, which explained both the influence of diffuse magnetic field on plasma and formation and mechanical stability of the flux tubes. In short, corona exists where pressure of the imbedded field gets comparable to plasma pressure.

Early stages of heavy ion collisions are believed to be described by the so called "glasma" , a set of random color fields created by color charges of partons of the two colliding nuclei at the 
moment of the collision [11]. For large nuclei those charges and fields can become large enough to be treated classically. However as two discs with charges move away from each other, those classical field are getting smaller and (in a still poorly understood process) rather quickly create the quark-gluon plasma. Transition from field to plasma has been subject of multiple works, too many to be mentioned. One notable idea is existence of instabilities [12] such as the so called Weibel instability, producing filamentation of the longitudinal flow and transverse magnetic field $B_{\perp}$. Asakawa et al [13] argued that such chaotic magnetic field would remain in plasma, leading to "abnormal viscosity".

Unfortunately those ideas, derived perturbatively and basically taken from electromagnetic plasma contexts, cannot possibly be valid for QGP at RHIC. The crucial difference between the QED and QCD plasmas lies in the existence of magnetically charged quasiparticles - monopoles and dyons - leading to nonzero magnetic screening mass $M_{M} \sim g^{2} T$ first suggested by Polyakov 30 years ago [14] and by now well confirmed by subsequent lattice studies. As at RHIC the central part of the produced fireball reaches relatively high temperature $T \sim 2 T_{c}$, we expect both $E, B$ fields to be effectively screened there, see the central cylindrical part of Fig.?? marked QGP.

Yet in the near- $T_{c}$ region surrounding the QGP, marked M (mixed or magnetic) in Fig.?? there will be surviving electric field, as explained above. A snapshot of the geometry of the $\mathrm{M}$ region at some early time is shown in Fig.??: here unscreened electric fields are shown by thin lines and magnetic flux tubes by think ones. The lower plot show similar snapshot at collision energy much smaller than at RHIC, planned to be investigated in a specialized run. It is very important to emphasize that although this region on the phase diagram is represented by a very narrow strip $\left|T-T_{c}\right| \ll T_{c}$, it corresponds to more than order of variation of the energy or entropy density, and the corresponding space-time volume in the expansion of the fireball is by no means small.

Magnetohydrodynamics is a well known part of plasma physics, developed by Alfven, Fermi, Chandrasekhar and many others since 1940's . It is an approximation which keeps only one (magnetic) field and half of Maxwell eqns, while the other (electric) is assumed to be totally screened. Ideal MHD approximation is the limit of infinite conductivity of plasma $\sigma \rightarrow \infty$, similar to zero viscosity approximation for ideal hydrodynamics. In MHD the coupling between the field and and matter is obtained by inclusion of the (magnetic) field contribution into the stress tensor of the medium.

Due to space limitations I will not put a complete set of resulting equations here, jumping directly to the main consequences. In a "(dual)magnetized" plasma small amplitude perturbations are split into two modes, known as Alfven waves, propagating with two different speeds

$$
u_{ \pm}^{2}=\left(\frac{1}{2}\right)\left[\frac{\vec{E}^{2}}{\varepsilon}+c_{s}^{2}\right] \pm\left(\frac{1}{2}\right)\left[\left(\frac{\vec{E}^{2}}{\varepsilon}+c_{s}^{2}\right)^{2}-\frac{4 \vec{E}^{2} \cos ^{2} \theta c_{s}^{2}}{\varepsilon}\right]^{1 / 2}
$$

where $E, c_{s}, \varepsilon$ are the electric field, the speed of sound in "unmagnetized" medium without field and plasma energy density, $\theta$ is the angle between the field strength and the the direction of the wave propagation. Note a case in which the wave goes transverse to the field $(\cos \theta=0)$ in which the lower mode has zero speed. In the case of jet quenching - when the jet direction is more or less up to experimentalist to pick - general shape of these waves can be complicated. However when the jet (or original charge fluctuation) propagates longitudinally, in the same direction as the field, the 
problem is axially symmetric and results in general in two cones. The angles of their propagation can be obtained from the previous expression, in which the 1.h.s. is substituted by Mach relation $u \rightarrow \cos (\theta) v, \mathrm{v}$ is the velocity of the jet, and solve it for the $\cos \theta$ (see the paper for more details on solutions).

If instead of electric field being constant in space one has a spot, localized in transverse plane, inevitably there is nonzero $\partial \vec{E}_{z} / \partial r$, which is a part of $\operatorname{curl}(\vec{E})$ and by the (dual) Maxwell equation it should be proportional to (dual, or magnetic) current $\tilde{j}_{\phi}$. This tells us that a flux tube solution must have a "coil" with a current running around and trying to cancel the field outside the spot. Ideal DMHD has axially symmetric solution - the macroscopic flux tube, in which field pressure can balance that of the plasma - similar to what is used in solar physics. We dont discuss them in detail here because we think the flux tubes appeared in QGP corona are actually microscopically thin, and so should be considered in a way discussed by Liao and myself previously [7].

\section{The ridges and the cone}

Three different correlation phenomena have been discovered in heavy ion collisions at RHIC:

(i) The "cone" has been discovered in the 2-particle azimuthal correlations, as the disappearance of the "away-side" peak at $\Delta \phi=\pi$ and appearance of new peaks at completely different angle, as one moves from peripheral to central collisions. After discovery, extensive studies of 2 and 3-particle correlations confirmed that the observed structure is indeed cone-like, and not e.g. a reflected jet. The cone angle is in the range $\theta=1.2-1.4$ radians (not too far from $\pi / 2=90^{\circ}$ or cylindrical waves!). The Mach formula gives the speed of pertinent perturbation to be about

$$
<v_{\text {wave }}>=\cos \left(\theta_{M}\right) \approx 0.2
$$

which is below the expected speed of sound (even near $T_{c}$ lattice gives about .3).

(ii) the hard ridge is also seen in 2-particle correlators plotted on the two-dimensional $\Delta \phi-\Delta \eta$ plane, the differences between the azimuthal angles and pseudorapidities of the two particles. The jet remnants make a peak near $\Delta \phi=0, \Delta \eta=0$, which sits on top of the "ridge", with comparable width in $\Delta \phi$ but very wide width $\Delta \eta$. For plots and various features one can consult the original talk by Putschke [16].

(iii) the "soft ridge" is found by STAR collaboration [17, 18] without a trigger, in the 2-particle correlations. For many experimental details and phenomenological considerations related to these phenomena the reader may consult e.g. the talks at recent specialized workshop [19]. Further confirmation of hydro origin of ridges comes from the centrality dependence of the angular width of the ridge: the peak in azimuth sharpens for more central collisions, see Fig.2. This happens because of two interrelated effects, both well documented. For central collisions there is (i) an increase of the radial hydro velocity, accompanied by (ii) a substantial decrease in the freezeout temperature (which goes from $T_{c} \approx 170 \mathrm{MeV}$ in peripheral down to $T_{f} \approx 90 \mathrm{MeV}$ for central collisions.

We will return to these observations below, turning now to their suggested explanations:

(i) Stoecker et al, as well as Casalderrey, Teaney and myself [20] have proposed that the energy deposited by a quenched jet goes into two hydrodynamical excitation modes, the sound and the so called diffusion or wake modes. The sound from the propagating jet should thus create the famous 
Mach cone, in qualitative agreement with the conical structure observed.

(ii) One early model for "hard ridge" has been introduced in my paper [21]. It relates it with the forward-backward jets accompanying any hard scattering, providing extra particles ("hot spot") widely distributed in rapidity. This idea is then combined with the one suggested previously by Voloshin [22], namely that extra particles deposited in the fireball would be moved transversely by the radial hydrodynamical flow, should produce a peak at certain azimuthal angle corresponding to the position of the hot spot, see Fig.??(a). While particles of the ridge are separated by large rapidity gaps and cannot communicate during the expansion process, their azimuthal emission angles remain correlated with each other because they originate from the same "hot spot" in the transverse plane.

(iii) Similarly, transverse hydro boost of "hot spots" was used for the explanation of the "soft ridge" by McLerran and collaborators [23, 24]. They have pointed out that the initial state color fluctuations in the colliding nuclei would create long-range rapidity correlations.

So, at a very qualitative level the origin of all three phenomena seem to be explained: yet at more qualitative level a lot of puzzles appear. As an example, consider the simplest of them, the "soft ridge". As discussed in [23, 24], the initial stage (proper time $\tau \sim 1 / Q_{s} \sim 0.2 \mathrm{fm} / \mathrm{c}$ where $Q_{s} \sim 1 \mathrm{GeV}$ is the so called saturation scale at RHIC) can be discussed using classical Yang-Mills equations: thus color fluctuations naturally appear. However, the observed pions come from final freezeout time, separated from the initial "glasma" era by much longer time $\tau \sim 10 \mathrm{fm}$. This is certainly so, as the explanation heavily relies on radial hydro velocity and thus it has to wait till the hydro velocity is being created. As we will argue below, there are many reasons why one might have expected nearly complete disappearance of this signal during this time.

Common to all three cases is deposition of some additional energy (or entropy), on top of the "ambient matter". The number of correlated particles in all of them constitute a small $(\sim$ $10^{-3}$ ) fraction of the total multiplicity: thus they can only be seen in a high-statistics correlation analysis. These perturbation would give rise to divergent conical (or cylindrical, or spherical) waves. Similar to circles from a stone thrown into a pond, initial perturbation may become some waves, with basically nothing left at the original location at later time. Even without dissipation, ideal hydrodynamics predicts that the final radius of those waves is given by the "sound horizon"

$$
R_{h}=\int_{0}^{\tau_{f}} d \tau c_{s}(\tau)
$$

As we will detail below, by the the freezeout proper time $\tau_{f} \sim 10-15 \mathrm{fm} / \mathrm{c}$, this distance is not small, $\sim 6 \mathrm{fm}$ or so, since the speed of sound changes between $c_{s}=1 / \sqrt{3} \approx .58$ in QGP and about .3 at its minimum near $T_{c}$ The amplitude of the wave is decreasing accordingly, and the width of $\phi$ distribution grows, making us wandering if any trace of the perturbation can remain observable. And yet, we do observe all three correlations, as if nothing happened to them!

The magnitude of the peak's width also provides a limit on how widely the "spot" can evolve, from its creation at initial time to the freezeout moment. In Fig.2 we have plotted the shape of azimuthal peak produced by (semi) circles of radii $1 . .6 \mathrm{fm}$. We have superimposed Hubble flow and calculated this angular distribution. As one can see from this figure, the width of the distribution grows - it is $0.57,0.56,0.69,0.76,0.83,0.89$ for the 6 curves shown. Moreover, the distribution shapes become very different from that observed, with two maxima shifted from $\phi=0$ (correspond- 
ing to direction of flow at two points at which the circle intersect the fireball boundary). Comparing such distribution with observations, e.g. their width with those shown in Fig.2, we concluded that for central collisions the radius of a spot at freezeout is restricted to be $R\left(\tau_{\text {freezeout }}\right)<3 \mathrm{fm}$ or about factor two smaller than the radius of the sound horizon with realistic speed of sound .
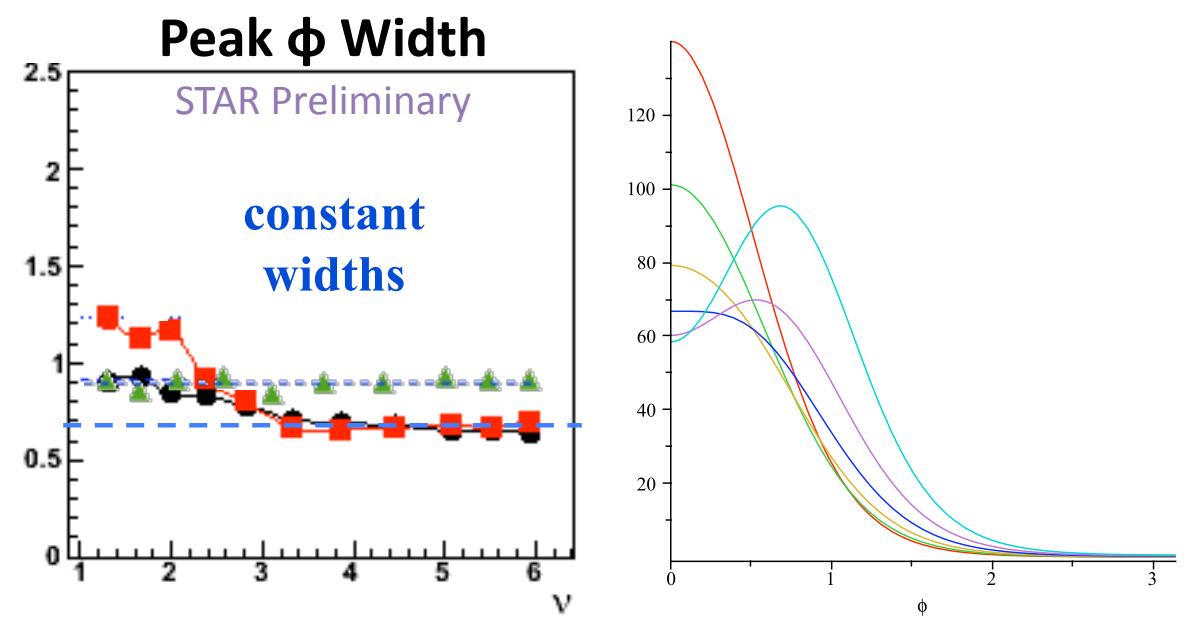

Figure 2: (left) Azimuthal width of the ridge as a function of centrality, from the talk of L.Ray [19]. The red squares and black dots are for 200 and $62 \mathrm{GeV} \mathrm{Au} \mathrm{Au} \mathrm{collisions.} \mathrm{Green} \mathrm{triangles} \mathrm{correspond} \mathrm{to} \mathrm{the} \mathrm{minijet}$ model without matter effects shown for comparison. (right) The dependence of the visible distribution in azimuthal angle on the width of the (semi)circle at the time of freezeout. Six curves, from the most narrow to wider ones, correspond to the radius of the circle 1,2,3,4,5,6 fm, respectively. The original spot position is selected to be at the edge of the nuclei. The distribution is calculated for particle of $p_{t}=1 \mathrm{GeV}$ and fix freezeout $T_{f}=165 \mathrm{MeV}$.

Why is it that we do not see expansion of the perturbations? Here the idea of the double cones/cylinders is used: it is the slower component, or even flux tubes completely stabilized by the pressure balance, can be the solution of the puzzle.

Thinking further along this line, let me point out some further consequences of the "corona" idea, which can be further tested in experiment. One direction is related with the predictions of the energy/centrality dependence of the ridges and cones. Already in the introduction we have shown in our sketch of the idea, depicted in Fig.1, we indicated that since the geometry of the M-phase domain is quite different for different collision energies. For RHIC the M-phase sits on the outside of the fireball (Fig.(a)), thus it get a maximal boost from the hydrodynamical expansion and may produce ridges with rather narrow peaks in the azimuthal angle. For much lower energies - corresponding both to the SPS fixed target experiments and planned RHIC scan down) - one should find the M-phase only on the inner part of the fireball (Fig.(b)), which experience little hydro boost if at all. The logical prediction is then that no soft or hard ridges should be observed in this case. Standard modelling using the realistic geometry/density distributions should make those predictions quantitative.

The "cone" is a different story: first of all, those do not rely on overall hydrodynamics and thus may well happen at the very center of the fireball and yet be observed. Second important distinction: "cones" are perturbations created by the "away-side" jet, and thus have completely 
different geometry/timing. If the trigger jet is surface biased, the away side jet has to fly through rather long path inside fireball, with its length varying roughly between its radius and diameter, 6-12 fm. As one can see from hydro solution, the M-phase starts at time zero at the edge and time about $5 \mathrm{fm} / \mathrm{c}$ at the center. Combining two observations together, one would see, that the away-side jet is travelling most of its long path in the M-phase. Furthermore, even at low collision energies, when the M-phase occupies only the central part of the fireball, this conclusion is still fulfilled. The prediction then is that "cones" should not show very strong dependence on collision energy and centrality, in contrast to ridges.

Let us now see how these ideas confront the available data. SPS experiments - CERES and NA49 - have not seen anything like ridges. The centrality and energy dependence of RHIC data do indeed suggest that this phenomenon is disappearing rather rapidly. On the other hand, both CERES and NA49 observe away-side structures which are remarkably similar to RHIC data on the away-side, see talk by Appelshauser at the workshop [19]. We conclude that the picture in which ridges originate from spacial part of the "QGP corona", while cones are from its temporal part, at times 5-10 fm, is in qualitative agreement with the data.

Finally, let me indicate two more directions of future studies. One is possible role of the unscreened electric fields in the M-phase in early-time hydro evolution. The pressure of the field, adding to (very low) pressure of the M-phase may help to start hydro a bit earlier and help explain the HBT puzzle. Some studies of the kind (but with non-equilibrium fields rather than DMHD ones) have been made in Ref. [25].

The second is related with recent RHIC data provided direct experimental indications for enhanced stability of the flux tube in matter relative to pp. We will use those from PHOBOS collaboration, which has large rapidity coverage of their silicon detector. It has been observed that the number of charged particles in a cluster in AuAu collisions is about twice that seen in pp collisions. The produced clusters are not isotropically decaying resonances, as in pp, , but are instead more extended in rapidity. This last fact shows a direct relation between clustering, the "soft ridge" and the flux tubes. Taken together, we interpret those clustering data as direct proof of significant changes in the flux tube decay parameters in AuAu relative to pp: the tubes apparently decay less frequently, into larger clusters, as we also argued above on the basis of theoretical (lattice-based) ideas.

\section{References}

[1] S. Mandelstam, Phys. Rept. 23, 245 (1976); G. ’t Hooft, “Topology Of The Gauge Condition And New Confinement Phases In Nonabelian Nucl. Phys. B 190, 455 (1981).

[2] J. Liao and E. Shuryak, Phys. Rev. C 75, 054907 (2007) [arXiv:hep-ph/0611131]. Phys. Rev. Lett. 101, 162302 (2008) [arXiv:0804.0255 [hep-ph]].

[3] M. N. Chernodub and V. I. Zakharov, Phys. Rev. Lett. 98, 082002 (2007) [arXiv:hep-ph/0611228].

[4] J. Liao and E. Shuryak, arXiv:0804.4890 [hep-ph].

[5] A. D’Alessandro and M. D'Elia, arXiv:0711.1266 [hep-lat].

[6] E. Shuryak, arXiv:0903.3734 [nucl-th].

[7] J. Liao and E. Shuryak, Phys. Rev. C 77, 064905 (2008) [arXiv:0706.4465 [hep-ph]]. 
[8] C. Ratti and E. Shuryak, arXiv:0811.4174 [hep-ph].

[9] A. Nakamura, T. Saito and S. Sakai, Phys. Rev. D 69, 014506 (2004) [arXiv:hep-lat/0311024].

[10] E. V. Shuryak and I. Zahed, Phys. Rev. D 70, 054507 (2004) [arXiv:hep-ph/0403127].

[11] L. D. McLerran and R. Venugopalan, Phys. Rev. D 49, 2233 (1994) [arXiv:hep-ph/9309289].

[12] S. Mrowczynski, Phys. Lett. B 314, 118 (1993).

[13] M. Asakawa, S. A. Bass and B. Muller, J. Phys. G 34, S839 (2007) [arXiv:nucl-th/0702007].

[14] A. M. Polyakov, Phys. Lett. B 72, 477 (1978).

[15] CATHIE workshop "Critical assessment of theory and experiments on correlations at RHIC, Feb.25-26, BNL. http://www.bnl.gov/cathie-riken

[16] J.Putschke, Talk at Quark Matter 2006 (for STAR collaboration), Shanghai, Nov.2006. J.Phys.G: Nucl.Part.Phys.34 (2007) S679

[17] J. Adams et al. [STAR Collaboration], J. Phys. G 32, L37 (2006) [arXiv:nucl-ex/0509030].

[18] M.Daugherity (for the STAR coll.), Anomalous centrality variation..., QM08, J.Phys.G.Nucl/Part.Phys. 35 (2008) 104090

[19] CATHIE workshop "Critical assessment of theory and experiments on correlations at RHIC, Feb.25-26, BNL. http://www.bnl.gov/cathie-riken

[20] J. Casalderrey-Solana, E. V. Shuryak and D. Teaney, J. Phys. Conf. Ser. 27, 22 (2005) [Nucl. Phys. A 774, 577 (2006)] [arXiv:hep-ph/0411315]. L. M. Satarov, H. Stoecker and I. N. Mishustin, Phys. Lett. B 627, 64 (2005) [arXiv:hep-ph/0505245].

[21] E. V. Shuryak, Phys. Rev. C 76, 047901 (2007) [arXiv:0706.3531 [nucl-th]].

[22] S. A. Voloshin, Phys. Lett. B 632, 490 (2006) [arXiv:nucl-th/0312065].

[23] A. Dumitru, F. Gelis, L. McLerran and R. Venugopalan, Nucl. Phys. A 810, 91 (2008) [arXiv:0804.3858 [hep-ph]].

[24] S. Gavin, L. McLerran and G. Moschelli, Phys. Rev. C 79, 051902 (2009) [arXiv:0806.4718 [nucl-th]].

[25] J. Vredevoogd and S. Pratt, arXiv:0810.4325 [nucl-th].

[26] G.S.F.Stephans (Phobos collaboration) talk at AGS/RHIC Users meeting, June 2009, see also Phys. Rev. C75(2007)054913.arXiv: 0812.1172 G. I. Veres et al. [PHOBOS Collaboration], arXiv:0806.2803 [nucl-ex]. 